EPJ Web of Conferences 81, 02013 (2014)

DOI: $10.1051 /$ epjconf/ 20148102013

(C) Owned by the authors, published by EDP Sciences, 2014

\title{
Polarization determination for the studies of the eta meson pro- duction
}

\author{
Iryna Ozerianska ${ }^{1}$, a , Paweł Moskal ${ }^{1}$, and Marcin Zieliński ${ }^{1}$ \\ for the WASA-at-COSY Collaboration \\ ${ }^{1}$ Institute of Physics, Jagiellonian University, 30-059 Kraków, Poland
}

\begin{abstract}
The dynamics of $\eta$ meson production and the interaction of $\eta$ mesons with nucleons can be studied using the $\vec{p} p \rightarrow p p \eta$ reaction via measurements of the analyzing power $A_{y}$. To this end, we have performed a measurement of the $\vec{p} p \rightarrow p p \eta$ reaction using the large acceptance and $\varphi$ symmetric WASA-at-COSY detector, for beam momenta of $2026 \mathrm{MeV} / \mathrm{c}$ and $2188 \mathrm{MeV} / \mathrm{c}$.
\end{abstract}

\section{Introduction}

Precise study of the interaction of the $\eta$ meson with a nucleon and the production mechanism requires measurements of the $A_{y}$ analysing power [1-4]. So far, a high statistics measurement of the $\vec{p} p \rightarrow p p$ reaction was made with the large acceptance and symmetric WASA detector [5] installed on the COSY accelerator. The measurement was conducted for beam momenta of $2026 \mathrm{MeV} / \mathrm{c}$ and $2188 \mathrm{MeV} / \mathrm{c}$ [6] which correspond to excess energies of $15 \mathrm{MeV}$ and $72 \mathrm{MeV}$, respectively. Proton ejectiles were registered in the forward part of the WASA detector, while the $\eta$ meson decay products (e.g. $\eta \rightarrow \gamma \gamma$ ) were detected in the central Electromagnetic Calorimeter. To monitor the degree of polarization, luminosity and the detector performance, simultaneously the $\vec{p} p \rightarrow p p$ reaction was measured. In order to control effects caused by the potential asymmetries in the detector setup, the spin of the proton beam was flipped for every accelerator cycle.

\section{Polarization determination}

The degree of polarization was determined based on the identification of events corresponding to elastically scattered protons according to the equation:

$$
P=\frac{1}{A_{y}} \cdot \epsilon\left(N(\theta, \varphi)^{\exp }, N(\theta, \varphi+180)^{\exp }\right)
$$

Asymmetry, $\epsilon$, was calculated as:

$$
\frac{N_{+}^{\exp }(\theta, \varphi)-N_{-}^{\exp }(\theta, \varphi)}{N_{+}^{\exp }(\theta, \varphi)+N_{-}^{\exp }(\theta, \varphi)}=\epsilon\left(N(\theta, \varphi)^{\exp }, N(\theta, \varphi+180)^{\exp }\right)
$$

a. e-mail: i.ozerianska@gmail.com 
and $N(\theta, \varphi)^{\exp }$ and $N(\theta, \varphi+180)^{\exp }$ denote number of events for each angular bin. The value of analyzing power $A_{y}$ was taken from independent EDDA measurement [7].

All the events were acceptance corrected, and the low acceptance region from 38-46 deg was rejected (see Fig. 1). The exemplary asymmetry plots are shown in Fig. 2 .
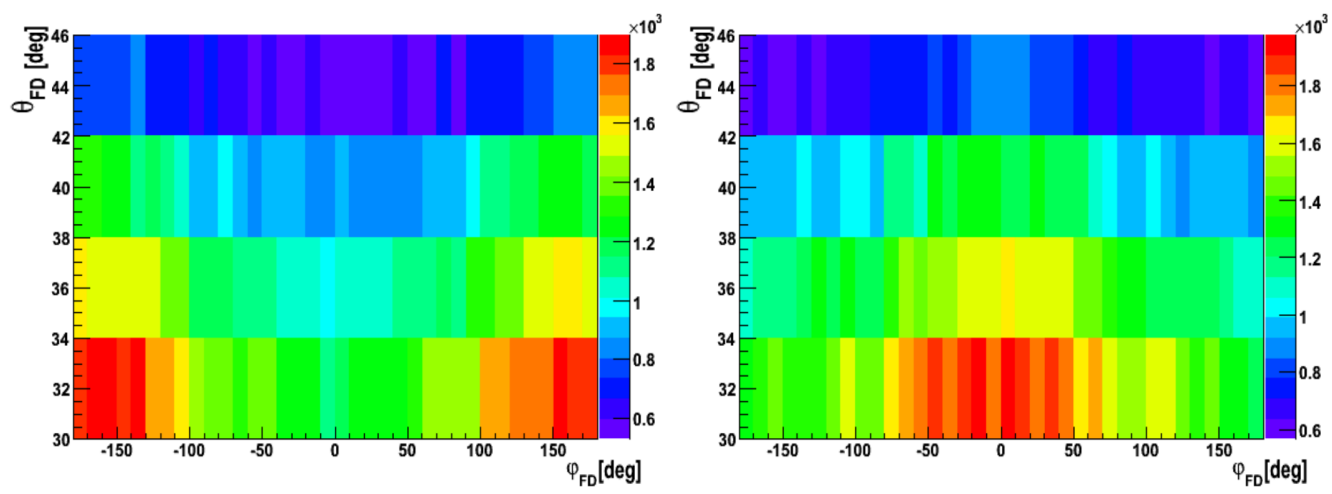

Figure 1: Example of the experimental distribution of the $\theta_{F D}$ as a function of the protons azimuthal angle.
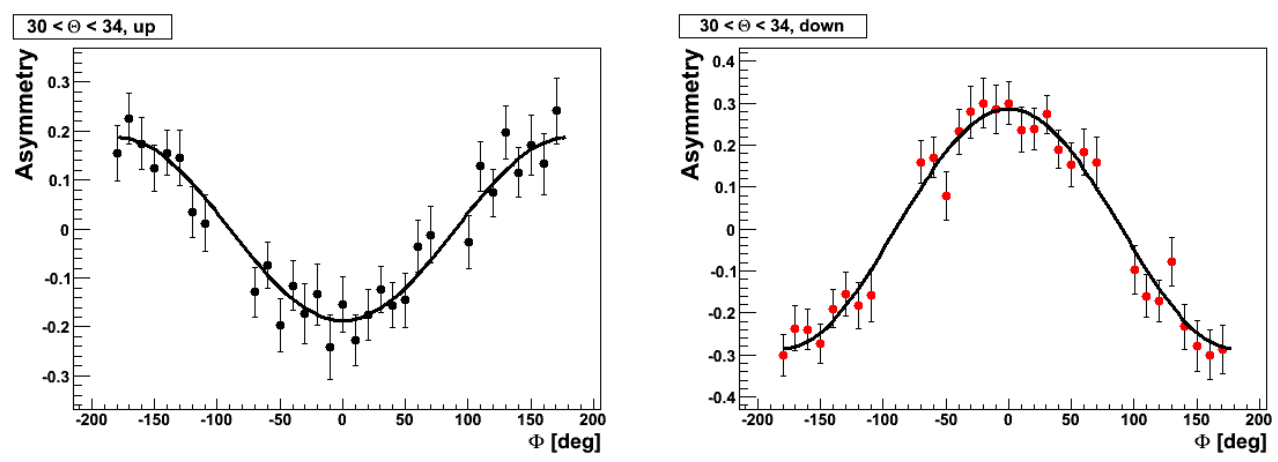

Figure 2: Experimental distribution of the asymmetry as a function of the protons azimuthal angle, plotted for proton scattering angles between $30^{\circ}$ and $34^{\circ}$. Black line represents the fit function.

For the precise determination of the polarization we have determined the coordinates of the experimental interaction point. To find the vertex coordinates, $\left(v_{x} ; v_{y} ; v_{z}\right)$, two methods $[8,9]$ were applied in the experiment. Results obtained from both methods are presented at Tab. 1.

Preliminary results for the degree of polarization, using the whole data sample and calculated based on the asymmetry determined for each experimental run separately, are presented in Fig.3. One can see that the polarization value varies not only for the opposite spin mode (up or down), but also for the different beam momenta $2026 \mathrm{MeV} / \mathrm{c}$ and for the $2188 \mathrm{MeV} / \mathrm{c}$. One explanation for such an effect is the depolarizing resonances which need to be crossed during the acceleration of the beam [10]. 
MESON 2014 $-13^{\text {th }}$ International Workshop on Production, Properties and Interaction of Mesons

\begin{tabular}{|c||c|c|c|}
\hline vertex & unpolarized $P_{\text {beam }}=2.026 \mathrm{Gev} / \mathrm{c}$ & $P_{\text {beam }}=2.026 \mathrm{Gev} / \mathrm{c}$ & $P_{\text {beam }}=2.188 \mathrm{Gev} / \mathrm{c}$ \\
\hline \multicolumn{4}{|c|}{ The $\chi^{2}$ method } \\
\hline$x_{v}$ & $-0.1164 \pm 0.0052$ & $-0.1230 \pm 0.0011$ & $-0.2834 \pm 0.0010$ \\
$y_{v}$ & $0.1119 \pm 0.0052$ & $0.1099 \pm 0.0011$ & $0.1551 \pm 0.0010$ \\
\hline \multicolumn{3}{|c|}{ The distance method } \\
\hline$x_{v}$ & $-0.0908 \pm 0.0017$ & $-0.0968 \pm 0.0012$ & $-0.3755 \pm 0.0019$ \\
$y_{v}$ & $0.1386 \pm 0.0019$ & $0.1369 \pm 0.0011$ & $0.1793 \pm 0.0015$ \\
\hline
\end{tabular}

Table 1: Vertex position calculated for the experiment based on the two independent methods. Detailed descriptions of the methods are given in [8,9].

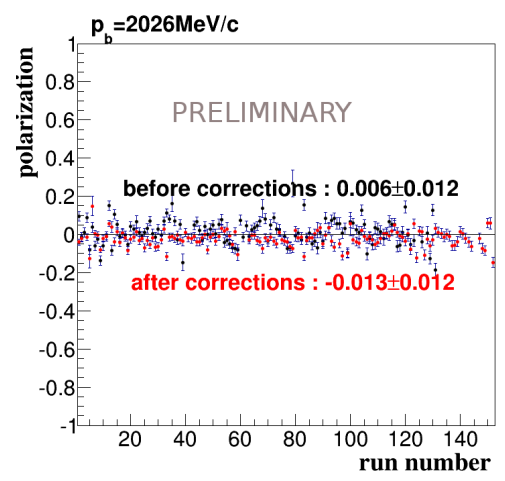

(a) unpolarized

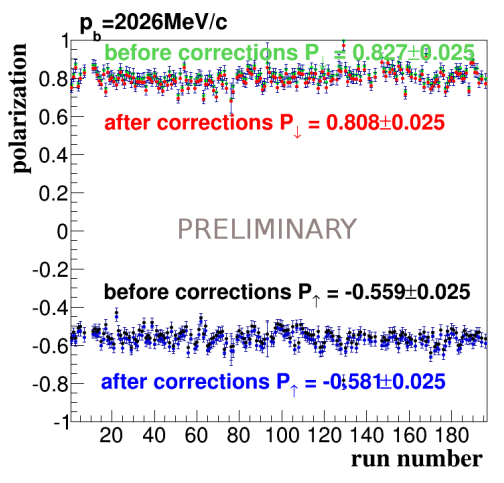

(b) polarized

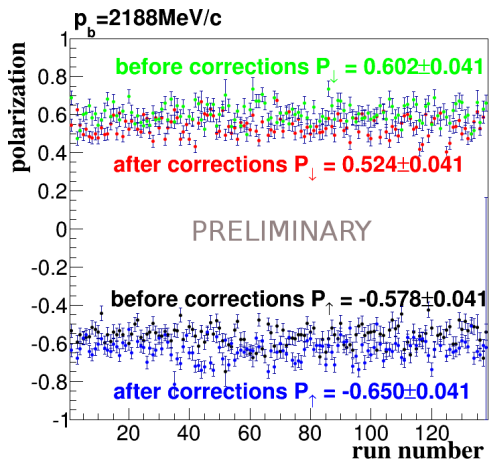

(c) polarized

Figure 3: Distribution of the polarization as a function of the run number, made for the proton's scattered into an angle between $30^{\circ}$ and $38^{\circ}$.

As a cross check we also calculated the polarization by adding all events together and determining the asymmetry for the whole data sample. As a result we have polarization for the elastic scattered events calculated with two methods, the differences can be used as systematic errors. 


\section{Outlook}

The goal of the studies is to determine $A_{y}$ for the $\vec{p} p \rightarrow p p \eta$ reaction. As a first step we have preselected the data in search for the $\eta$ meson and to determine the number of recorded mesons we have reconstructed the missing mass for the reaction $p p \rightarrow p p X$. Fig. 4 shows the preliminary result of distribution of the missing mass of two protons measured in coincidence in the Forward Detector for the $30 \%$ of collected data.

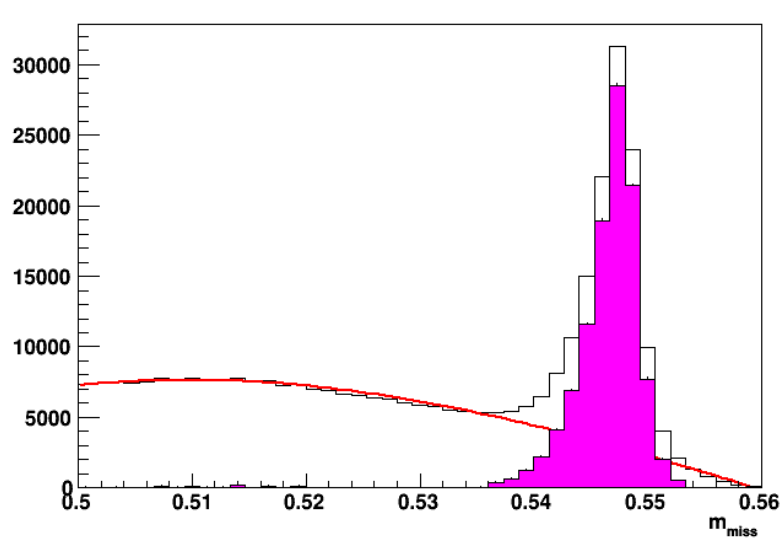

Figure 4: Distribution of the missing mass of two protons in the reaction $p p \rightarrow p p X$.

\section{Acknowledgements}

We acknowledge support by the Polish National Science Center through grant No. 2011/03/B/ST2/01847, by the FFE grants of the Research Center Jülich, by the EU Integrated Infrastructure Initiative HadronPhysics Project under contract number RII3-CT-2004-506078 by the European Commission under the 7th Framework Programme through the 'Research Infrastructures' action of the 'Capacities' Programme, Call: FP7 - INFRASTRUCTURES - 2008 - 1, Grant Agreement N. 227431, and by the Polish Ministry of Science and Higher Education through grant No. 393/E-338/STYP/8/2013.

\section{References}

[1] P. Winter et al., Phys. Lett. B544 (2002) 251-258

[2] R. Czyżykiewicz et al., Phys. Rev. Lett. 98 (2007) 122003.

[3] F. Balestra et al., Phys. Rev. C 69 (2004) 064003.

[4] P. Winter et al., Eur. Phys. J. A 18 (2003) 355.

[5] H. H. Adam et al., nucl-ex/0411038.

[6] P. Moskal, M. Hodana, J. Phys. Conf. Ser. 295 (2011) 012080; e-Print: arXiv:1101.5486.

[7] M. Altmeier et al., Phys. Rev. Lett. 85 (2000).

[8] M. Hodana, P. Moskal, I. Ozerianska and M. Zieliński, Acta Phys. Pol. B 45 (2014) 697.

[9] M. Hodana, P. Moskal, I. Ozerianska, Acta Phys. Pol. B Suppl. 6 (2013) 1041.

[10] R. Gebel, private communication, 2014. 